prescriptions were judged to be appropriate. The mean $\mathrm{PaO}_{2}$ on blood gas samples was $13.1 \mathrm{kPa}$ compared with $15.1 \mathrm{kPa}$ in 2005 and $14.9 \mathrm{kPa}$ in 2010. Mean $\mathrm{PaCO}_{2}$ was $5.3 \mathrm{kPa}$. The mean $\mathrm{SpO}_{2}$ (pulse oximetry) was 96.8\% [median 97\%, range $91 \%-100 \%$ ]. $82 \%$ of $\mathrm{SpO}_{2}$ values were within the target range but four of six patients with target range 88\%-92\% were at least $2 \%$ above this range. Attitudes and practice in our Critical Care Unit have changed in the past decade and hyperoxaemia is less common now. However, practice still lags behind the declared ambition of our Critical Care colleagues to maintain normoxaemia for most patients. We have instituted changes to CCU practice in May-June 2017. These changes will inform the design of a systematic randomised cluster implementation study using a step-wedge design to implement current best practice in a wide range of Critical Care units.

\section{P130 AUTOMATED VIDEO MONITORING OF SPONTANEOUS BREATHING RECOVERY DURING THE HIGH FREQUENCY JET VENTILATION}

${ }^{1} \mathrm{MB}$ Kontorovich, ${ }^{2} \mathrm{KS}$ Purtov, ${ }^{2} \mathrm{VS}$ Kublanov. 'Regional Anti-Tuberculous Dispensary, Yekaterinburg, Russia; ${ }^{2}$ Ural Federal University, Engineering School of Information Technologies Telecommunications and Control Systems, Yekaterinburg, Russia

\subsection{6/thoraxjn-2017-210983.272}

Introduction Automatic evaluation of spontaneous breathing recovery for patients during artificial ventilation is one of the central problems in the early postoperative period. The basic criteria for adequate breathing recovery are rhythmic movements and respiration muscle tone. The current paper presents the possibilities of using video processing technology to determine spontaneous breathing recovery in patients during high frequency jet ventilation (HFJV). We refer to this technology as remote body plethysmography(rBPG).

Materials and Methods The 16 subjects (male and female) involved in the experiment, aged between 24 and 76, had undergone operation of the thoracic cavity. Each patient provided written informed voluntary consent prior to study procedures. Immediately after operation, patients enter the intensive care unit, and have HFJV administered for a time between $30 \mathrm{~min}$ up to 2 hours or until the full recovery of muscle tone, consciousness and adequate spontaneous breathing is made. The HFJV was performed by the ZisLine JV100A device (Triton Electronics Systems Ltd., Russia, registration No 2010/08739). The reference respiration rate signal was measured by impedance pneumography with an MP 6-03 monitor (Triton Electronics Systems Ltd., Russia, registration N2007/00597). The patient body video recording was performed at a distance of $80 \mathrm{~cm}$ using two Logitech C920 webcams with $640 \times 480$ pixel resolution and $30 \mathrm{~Hz}$ sampling frequency. The original video processing software was used to rBPG signals assessment in real-time. The chest and epigastric movements were processed independently.

Results The Results of rBPG measurement showed that in most cases the process of restoring spontaneous breathing begins with diaphragmatic breathing. The thoracic breathing recovery can be quantified through the measurement of chest movement amplitude. The example of breathing recovery presented in figure 1 . The amplitude is raised alongside muscle tone recovery.

Conclusion rBPG provides readings of measurements of diaphragmatic and thoracic breathing from epigastric and chest regions. It allows the relative contribution of each region in total respiration to be quantified. Thus, rBPG can be used for assessing respiration muscle tone recovery and for measuring respiration parameters. It can be used to accurately select the appropriate time for turning off the ventilator and for extubation.

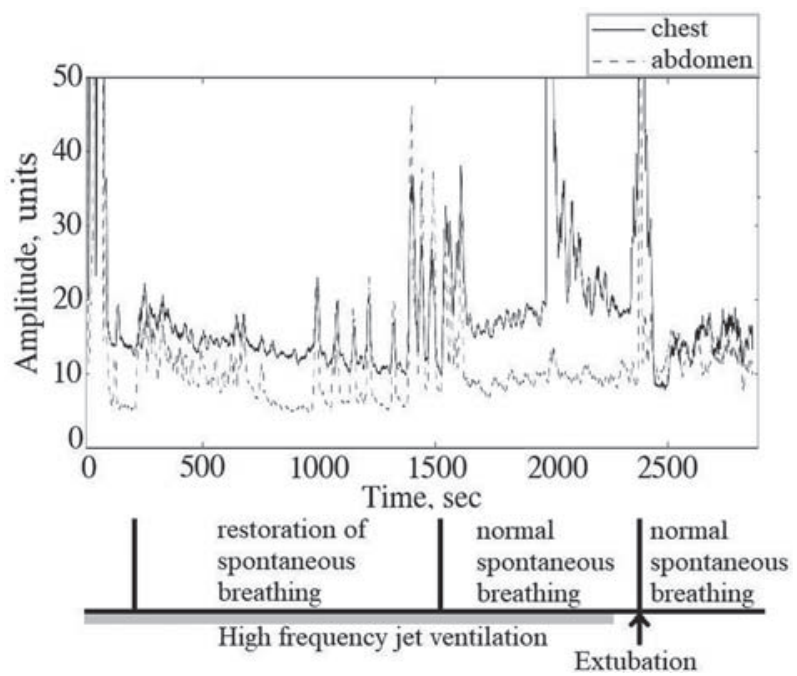

Abstract P130 Figure 1 The chest and abdomen movement amplitude alongside spontaneous breathing recovery.

\section{P131 WEANING OUTCOMES FROM TRACHEOSTOMY VENTILATION IN AN ACUTE RESPIRATORY CARE UNIT (ARCU): A THREE-YEAR EXPERIENCE}

S Sufyan, MN Khan, M Thirumaran, SP Meghjee, AOC Johnson, A Dwarakanath. Mid Yorkshire Hospitals NHS Trust, Wakefield, West Yorkshire

\subsection{6/thoraxjnl-2017-210983.273}

Introduction Patients who had tracheostomy in intensive care unit (ICU) as part of acute admission and are slow to wean from ventilation are admitted to our acute respiratory care unit (ARCU). We evaluated the long-term outcomes of attempted weaning from ventilator support in terms of underlying diagnosis, comorbidities, length of stay (LOS), level of support at discharge and one year survival.

Methods Twelve patients admitted to ARCU as a step-down from ICU between January 2014 and December 2016 were included. Patients were identified using discharge database and data was collected from electronic records and patient notes. Patients were excluded if they had tracheostomy inserted on a previous admission.

Results The patient demographics, length of stay on ARCU and primary diagnosis leading to respiratory failure requiring intubation and subsequent tracheostomy and the LOS on ICU and ARCU are described in Table 1. All but two had significant other comorbidities including neuromuscular disorders, COPD, cardiovascular disorders and OSA. No patients died in hospital. Eight $(67 \%)$ patients were discharged without any ventilatory support after decannulation, Two (17\%) required overnight ventilation and were discharged with tracheostomy ventilation. One patient was transferred to the neuro rehabilitation unit and one to a different ARCU with tracheostomy (self ventilating). Complications during weaning included pneumonia, pneumothorax, delirium, persistent secretions/mucus 